\title{
Individual Surgeon Outcomes and the Public
}

\author{
Peter T. McCollum ${ }^{1}$
}

Published online: 24 April 2017

(C) Association of Surgeons of India 2017

The disclosure of all individualised surgical outcomes within vascular surgery has raised a number of contentious issues. There were considerable political pressures applied to organisations and individuals to persuade surgeons to release their personal outcome data, and in 2014 within the vascular speciality, only six consultants eventually did not agree to their data being released. Subsequently, the government has dictated that everyone must agree to their data being released, despite the obvious accuracy issues.

In order to set a "honey trap", in 2014, I originally refused to allow my own personal data to be published. This was so that the media would potentially come to me to establish why I would not release my data and to allow me to explain the problem and difficulties with the data. This was remarkably successful in its aims and I was interviewed by national radio and television! From 2015, vascular surgeons were not allowed to opt out from the publication of individualised results despite there being some uncertainty about the legal position in terms of the Data Protection Act from our " $m$ 'learned friends" at the legal bar!

So what have been the problems? The principle of transparency is, of course, a good one but transparency in the context of outcome data must mean that the data itself is reliable, complete, understandable and relevant. Unfortunately, the aortic aneurysm mortality data published at an individual level failed in the UK on all levels. Although many individuals and many centres submitted their complete data set, a large

Peter T. McCollum

profpetermccollum@gmail.com

1 Department of Surgery, King Abdulaziz Medical City, NGA, Jeddah, Kingdom of Saudi Arabia number of centres and individuals originally did not and many still do not. It is quite clear that they were most likely to have avoided entering those patients who had a less successful outcome into the National Vascular Registry (NVR).

In order to achieve anything like reasonable numbers, elective open aneurysm repairs and endovascular aneurysm repair outcomes have had to be combined. The expected and, indeed, actual mortality for each of these groups is quite different and should be reported separately. Unfortunately, the average number of elective aneurysms (open and EVAR) performed over the first five-year period was only 30 per individual consultant, and meaningful analysis of mortality on the basis of such small numbers is almost impossible. During the same period, I performed 129 aneurysm repairs; 111 were open, which was one of the largest numbers in the UK for an individual. For what it is worth, my risk-adjusted mortality in 2014 was $1.7 \%$ rising to 2.6 in 2015 . However, even this number is inadequate to determine whether I am a "good" or a "bad" surgeon.

So what of the media? The BBC were actually originally extremely fair in their approach and presented a very balanced viewpoint, recognising that there were significant issues with the data and that statistically no real conclusions could be really be drawn. The newspapers were and still are less concerned with a balanced approach and at least one again published a list of surgeons who were at the bottom of the national "league table" even though there was no statistical evidence to prove that they were poor surgeons. This was completely unacceptable and essentially made it exceptionally difficult for named surgeons to carry on with their aortic aneurysm practice. Equally importantly, it has to be noted that there may well have been "poor" vascular surgeons within the wider data who were and are unidentified because the numbers of operations performed over a five-year period was inadequate to judge whether they were good or bad. This is in contrast to 
coronary artery bypass surgery, where individual surgeons do $100+$ cases per year, and thus, individualised surgeon mortality data is at least more meaningful.

The unintended consequences of publication of individualised outcomes are fairly apparent to the informed reader. There is a real risk of making the "acceptable" mortality so low as to be effectively unachievable. Importantly, young consultants are extremely unlikely to want to take on high-risk cases. With the increasing enthusiasm for endovascular repair, the cases left for open repair are becoming more complex and present a higher risk. Who would want do these in our brave new world?

An alternative, more informed approach is needed. At least within vascular surgery, the way forward is for us to concentrate on developing a smaller number of large units where the caseload is sufficient to be able to judge outcome, at least at unit level. This probably means reducing the total number of vascular centres in the UK down to perhaps 50 major centres. Outcome data should then be assessed at unit level, where there is sufficient internal peer pressure to ensure that individuals are performing at a reasonable level. We are all jealous of our individual unit performance. As numbers of cases dwindle, there may even be a case for a selected number of surgeons concentrating on open repair. Further, there is more opportunity for dual consultant operating with added patient safety benefit and the passing on of skills.
The implications for other surgical specialities are varied. Many, such as breast surgery, cannot use mortality as a reasonable marker of outcome. Others will find themselves in the same position as vascular surgery where (for instance) individualised mortality data in oesophageal surgery will run into the same problems that aortic aneurysm surgery has seen. Again, large units and unit-based data are probably the best way forward here. Other speciality groups will need to be clear as to what end-points should be used for outcome reporting within their individual specialities. Importantly, there is an urgent need for units to collect and collate their data honestly and submit it to their national databases, where one exists. Such data collection and collation inevitably requires resource but sadly the NHS has been very slow indeed to provide support for individual consultants or units to help them perform this important role. Where support exists (as in cardiothoracic surgery), the compliance with data collection is generally excellent, and as a result, meaningful conclusions can often be drawn.

What is the relevance of these data for India? At present, there is no "big brother" looking over a surgeon's shoulder in India at present but times change quickly.

The public deserve to see information about their surgeon but the raw data must be robust, reliable and relevant. After all, true information is data which has been interpreted by experts. 